\title{
Instant messenger smartphone application for endosonographer| cytopathologist real-time interaction at a distance in EUS-FNA for solid pancreatic lesions
}

(๑) $\circledast \ominus$

\author{
Authors \\ Angelo Ferrari Jr. ${ }^{2}$, Ermelino Libera ${ }^{2}$ \\ Institutions \\ 1 Department of Pediatrics, Escola Paulista de Medicina/ \\ UNIFESP \\ 2 Department of Medicine, Division of Gastroenterology, \\ Escola Paulista de Medicina/UNIFESP \\ 3 Department of Pathology, Escola Paulista de Medicina/ \\ UNIFESP
}

Rodrigo S. Machadoํㅜ, Rafaela Richa², Fabiano Callegari³ ${ }^{3}$ Giovana B. Souza², Luciano L. Tolentino², Frank S. Nakao²,

submitted 22.1.2019

accepted after revision 29.5.2019

\author{
Bibliography \\ DOI https://doi.org/10.1055/a-0965-6631 | \\ Endoscopy International Open 2019; 07: E1027-E1030 \\ (c) Georg Thieme Verlag KG Stuttgart · New York \\ eISSN 2196-9736
}

Corresponding author

Rodrigo S. Machado, Universidade Federal de Sao Paulo Escola Paulista de Medicina - Pediatrics, Av Onze de Junho 678, apto 26 Sao Paulo SP 04023-062, Brazil

Fax: +551155764344

rodrigo.strehl@gmail.com

\section{ABSTRACT}

Background and study aims Telecytopathology (TCP) may allow proper and timely evaluation of endoscopic ultrasound-guided fine-needle aspiration (EUS-FNA) specimens. The aim of this study was to evaluate the feasibility of TC through a multiplatform instant messenger smartphone application to evaluate specimens of EUS-FNA of pancreatic solid lesions.

Patients and methods Twenty-three patients (14 male/9 female; median age: 56 yr.; age range: 33 - 86) with a solid pancreatic lesion were included. Exclusion criteria were as follows: age $<18$ yr and predominantly cystic lesions. During each EUS-FNA, after each pass, the aspirated material was spread over a glass slide and was stained by the endoscopist. The glass slide was then reviewed on a microscope with a smartphone fitted in, and the most representative fields were captured and sent to the cytopathologist using WhatsApp Messenger.

Results In initial evaluation using TCP rapid on-site evaluation (ROSE), adequate cellularity of the glass slide was detected in 16 of 23 patients (69.6\%). An initial diagnosis of malignancy (positive or suspicious) was possible in 14 of 23 patients (60.8\%).

Conclusion The current study demonstrated the feasibility of a low-cost, Internet-based, telecytopathology system using WhatsApp Messenger to provide ROSE of EUS-FNA slides in patients with solid pancreatic lesions.

\section{Introduction}

Endoscopic ultrasound-guided fine-needle aspiration (EUSFNA) of solid lesions close to the upper gastrointestinal tract is a well-established diagnostic tool. Rapid on-site evaluation (ROSE) is immediate on-site cytologic examination of the specimen, determining adequacy and allowing proper intervention to enhance tissue acquisition [1]. Although ROSE increases diagnostic accuracy, its incorporation into clinical routine is hindered by the cost of an available on-site cytopathologist.

Telemedicine is defined as use of communication networks to deliver healthcare services from one geographical location to another, while telepathology is defined as a form of communication between medical professionals that includes transmission of pathology images and associated clinical information for various clinical applications [2,3]. ROSE with telecytopathology (ROSE/TCP) is a promising alternative to conventional ROSE, allowing remote slide evaluation, but it requires dedicated hardware and specific software, including a capture system. WhatsApp Messenger is a multiplatform mobile phone application that allows users to send photos, documents and texts without significant loss of image quality [4]. Recent studies have shown the potential use of this capability in healthcare $[5]$. 
The study was designed to evaluate the feasibility of ROSE in EUS-FNA of pancreatic solid lesions aided by a cytopathologist at a distance receiving smear slides sent through WhatsApp of assuring adequacy of the aspirate.

\section{Patients and methods}

\section{Patients}

Twenty-three patients (14 male/9 female; median age: 56 yr.; age range: $33-86 \mathrm{yr}$ ) were evaluated over an 18-month period (June 2016 to October 2017). Inclusion criteria were EUS-FNA to investigate the pancreatic solid mass according to previous radiological examination. Informed consent was signed by all patients. Exclusion criteria were an age less than 18 years, a cystic mass, and an extrapancreatic lesion. If a patient had more than one EUS-FNA to evaluate the same mass, only the first one was included.

\section{EUS-FNA}

All FNA biopsies were performed under EUS guidance using a linear echo-endoscope (EG-530 UT2; Fujinon, Saitama, Japan). Briefly, after sedation, at the discretion of the endoscopist, the gastric and duodenal windows were examined, and the best track for puncture was chosen after careful examination using the Doppler mode. Next, EUS-FNA was performed (22-Gauge; Expect; Boston Scientific, Natick, Massachusetts, United States) under real-time echographic visualization, moving the needle back and forth inside the lesion 10 to 15 times. At the first pass, no suction was applied. If more passes were performed due to the lack of material, alternative techniques, such as vacuum and fanning, were employed.

After the puncture, the smear was spread over a glass slide using another glass slide and then was air-dried and stained with Panoptic Stain by a senior member of the endoscopic team (FSN). The stained slide was then reviewed immediately on a microscope fitted with a smartphone (Samsung Galaxy S7, Camera 12 Megapixels, Android 7) with 4G Internet access. Digital zoom was used to capture slide images. The microscope (Motic RBA400 EF-UP, Shanghai, China) had objectives from $\times 4$ to $\times 100$ ( $>$ Fig. 1 ).

The slide was thoroughly reviewed in the lower power objective by the endoscopist, and all areas with nucleated cells in the slide were further examined in the $\times 40$ objective, photographed, and sent by WhatsApp Messenger for Android to the pathologist (FMC) along with relevant clinical data. The pathologist provided feedback on the adequacy of the material and a first impression on diagnosis over the same application. Additional passes were conducted as needed according to this evaluation. Clinical data and pictures were deidentified, labeled only by date and order.

Finally, specimens in excess were placed in formaldehyde $10 \%$ for cell block evaluation and were sent to the pathology laboratory, as well as the slides. The pathological report was categorized as nondiagnostic, negative for malignancy, atypical/ suspicious or positive for malignancy. Nondiagnostic was defined by aspirates with only blood or gastrointestinal epithelial cells. This information was compared with the final pathologic

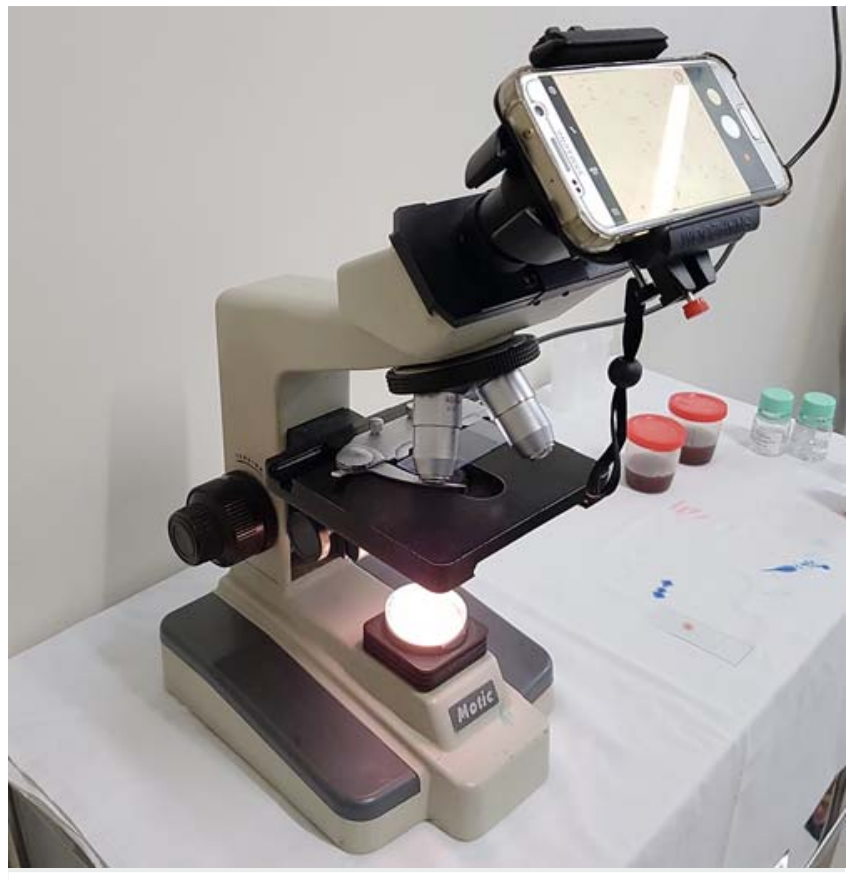

- Fig. 1 Microscope fitted with an Android smartphone for smear evaluation and image capture.

report (both questions), and the final diagnosis was dichotomized as malignant (malignant and suspicious) or nonmalignant (nondiagnostic and benign).

\section{Statistical analysis}

Qualitative variables were described by their proportion, while quantitative variables were described by their median and interquartile range (IQR). Concordance of adequacy between the preliminary evaluation and final pathological report (FPR) was estimated using the kappa coefficient. Sensitivity and specificity, as well as their respective $95 \%$ confidence intervals $(95 \% \mathrm{Cl})$, for diagnosis of malignancy were calculated. The gold standard for malignant disease was either a surgical histopathological report or a 12-month follow-up. Patients who were not followed at our outpatient clinic were surveyed by telephone.

Fisher's exact test was used to compare qualitative variables, and quantitative variables were compared with Student's $t$ test. In all tests, $P<0.05$ was regarded as significant. All statistical tests were conducted using $\mathrm{R}[6]$.

\section{Results}

ROSE/TCP showed adequate samples in 16 of 23 patients (69.6\%). Among the seven patients with inadequate samples, four were thereafter diagnosed with a malignant lesion after cell block evaluation. In > Table 1, the initial ROSE evaluation and final pathological report are depicted. Malignancy was the final diagnosis in 18 patients, among whom 14 had ductal adenocarcinoma, one had neuroendocrine tumor, two had metastatic tumors and one had a gastrointestinal stromal tumor 
- Table1 Diagnostic categories in the rapid on-site/telecytopathology (ROSE/TCP) evaluation and final pathology report.

\begin{tabular}{|c|c|c|c|c|c|}
\hline \multirow[t]{2}{*}{ ROSE/TCP } & \multirow[b]{2}{*}{ Non-diagnostic } & \multicolumn{2}{|c|}{ Final pathology report } & \multirow[b]{2}{*}{ Positive } & \multirow[t]{2}{*}{ Total } \\
\hline & & Negative & Suspicious & & \\
\hline Non-diagnostic & 3 & 0 & 0 & 4 & 7 \\
\hline Negative & 0 & 2 & 0 & 0 & 2 \\
\hline Suspicious & 0 & 0 & 1 & 2 & 3 \\
\hline Positive & 0 & 0 & 0 & 11 & 11 \\
\hline Total & 3 & 2 & 1 & 17 & 23 \\
\hline
\end{tabular}

- Table 2 Overall characteristics of pancreatic solid lesions and endoscopic ultrasound-guided fine-needle aspiration.

\begin{tabular}{|l|c|}
\hline Variables & Median (IQR) \\
\hline Size of lesion $(\mathrm{mm})$ & $48(28-57.5)$ \\
\hline Location & $15(65.2 \%)^{1}$ \\
\hline - Head of pancreas & $6(26.1 \%)^{1}$ \\
\hline - Body & $2(8.7 \%)^{1}$ \\
\hline - Tail & $9(39.1 \%)^{1}$ \\
\hline Duodenal window ${ }^{2}$ & $3(2-3)$ \\
\hline Number of passes & $8(5.5-10)$ \\
\hline Number of pictures & $1(4.3 \%)^{1}$ \\
\hline Need to repeat procedure & $18(78.3 \%)^{1}$ \\
\hline Final diagnosis of malignancy & \\
\hline $\begin{array}{l}\text { IQR, interquartile range } \\
1\end{array}$ & \\
\hline 2 in one patient, both gastric and duodenal windows were used.
\end{tabular}

(GIST-primary from pancreas). An initial diagnosis of malignancy, through TCP, was possible in 12 of 18 patients (66.7\%) with a final diagnosis of malignancy. The agreement coefficient after grouping negative vs. positive results was kappa coefficient $=0.6$. Overall sensitivity and specificity for diagnosing malignancy were, respectively, $100 \%$ (95\% Cl 82.4-100) and $100 \%$ (95\% Cl: 56.5-100).

The median number of pictures sent through the messenger was eight (interquartile range: $5.5-10)$ ( $>$ Table 2 ). Quality of pictures was regarded as satisfactory ( $>$ Fig. 2 ). In two patients, no picture was sent, as there was only blood in the slide after three and four FNA passes, respectively. Cell block evaluation revealed normal pancreatic cells in both patients, and the final pathologic report was not diagnostic, but there was no malignancy in the follow-up. Another patient with a nondiagnostic final pathologic report had two pictures sent, and the followup ruled out malignancy.

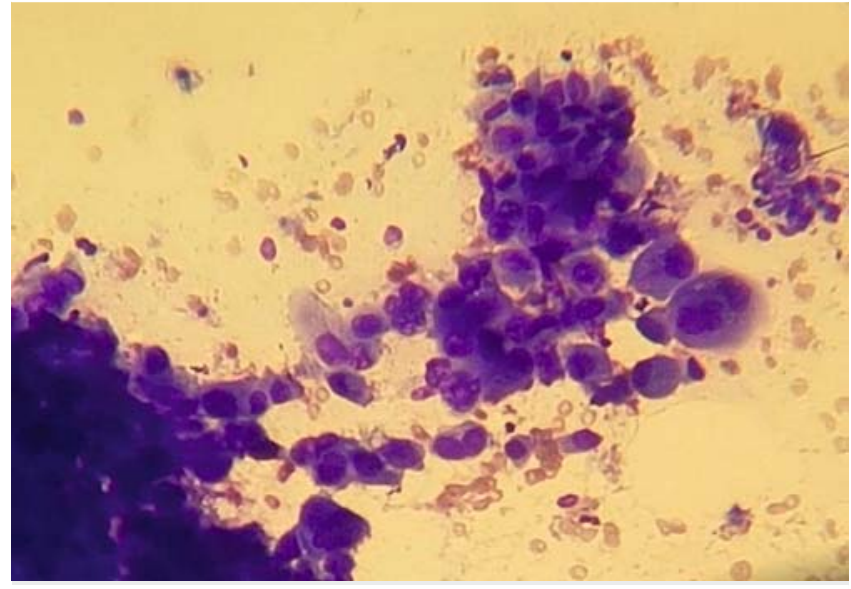

Fig. 2 Screenshot of WhatsApp messenger image from a specimen of a patient with a pancreatic adenocarcinoma.

\section{Discussion}

The current study demonstrated that cytopathological evaluation of pictures sent through a smartphone communication application from slides that were prepared by the endoscopist is feasible, possibly enhancing availability of ROSE. It allows proper evaluation of EUS-FNA specimens to determine adequacy of the sample as well as an initial diagnostic impression in some patients. Several studies have demonstrated successful use of telecytopathology for preliminary diagnosis of FNA specimens, with a dedicated on-site cytotechnologist and a proprietary system to deliver images to the pathologist $[7,8]$. The current study is the first to use an inexpensive smartphone-based application to deliver the images of cytopathological slides prepared by the endoscopist.

The system employed in the current report is a static telepathology system, as opposed to the dynamic ones, in which the pathologist can evaluate real-time images [9]. Well-known barriers to adoption of telepathology have included the cost of implementing and operating the technology, information technology-related challenges, pathologist perception of inferior performance relative to light microscopy, insufficient speed of these systems for high-volume clinical environments, as well as 
regulatory and licensure issues [3]. The advantages of the system that is herein presented are that it does not require proprietary equipment or software, and it uses a regular cell-phone 4G Internet connection, not dependent upon the hospital network. The smartphone messenger also has the potential to guide tissue acquisition for ancillary techniques, such as immunohistochemistry.

WhatsApp messenger has been increasingly reported in many ways as a tool to deliver health care, from evaluation of radiographic images to improving communication between health care providers and patients [4,5]. There are concerns related to confidentiality and privacy, but the messages are encrypted by the platform, and they are not stored in a cloud. In addition, the information was deidentified before being sent. Therefore, we believe that the app is a reliable tool, but its use should be locally regulated to avoid inappropriate leaking.

The study presented some important limitations. First, reproducibility was not assessed because only one cytopathologist and one EUS team participated. Some authors validated the new routine with archived material, but it was not possible because ROSE was implanted along with the TCP system [7]. Finally, the smears were prepared by the main endosonographer, who was trained in slide preparation. This routine has been used by other groups, with good results, including increased accuracy of EUS-FNA $[10,11]$.

The number of nondiagnostic procedures was 3 (13\%), and it did not impact overall accuracy $(100 \%)$ because those patients did not present with malignant disease. A previous study reported on ROSE with slides prepared by a cytotechnologist and a video microscopy system on 95 patients who had undergone EUS-FNA for pancreatic lesions compared with the nonROSE group. ROSE was associated with significantly fewer nondiagnostic procedures (3.7\% vs. $25.6 \%, P<0.0001$ ) [12]. On the other hand, Khurana et al. [13] reported that telecytopathology yielded an accuracy similar to that observed during conventional ROSE (84\% vs. $87 \%)$.

\section{Conclusion}

The current study demonstrated the feasibility of a low-cost, Internet-based, telecytopathology system using WhatsApp Messenger to provide ROSE of EUS-FNA slides in patients with solid pancreatic lesions. The application provides a low-cost alternative to conduct telecytopathology. Further studies are required to evaluate the reproducibility and overall feasibility in different settings.

Competing interest

None
References

[1] Hebert-Magee S, Bae S, Varadarajulu S et al. The presence of a cytopathologist increases the diagnostic accuracy of endoscopic ultrasound-guided fine needle aspiration cytology for pancreatic adenocarcinoma: a meta- analysis. Cytopathology 2013; 24: 159-171

[2] Sood S, Mbarika V, Jugoo S et al. What is telemedicine? A collection of 104 peer-reviewed perspectives and theoretical underpinnings Telemed J E Health 2007; 13: $573-590$

[3] Evans A], Krupinski EA, Weinstein RS et al. 2014 American Telemedicine Association clinical guidelines for telepathology: Another important step in support of increased adoption of telepathology for patient care. J Pathol Inform 2015; 6: $13-15$

[4] Giordano V, Koch H, Godoy-Santos A et al. WhatsApp Messenger as an adjunctive tool for telemedicine: an overview. Interact J Med Res 2017; 6: e11

[5] Giordano V, Koch HA, Mendes $\mathrm{CH}$ et al. WhatsApp messenger is useful and reproducible in the assessment of tibial plateau fractures: Inter- and intra-observer agreement study. Int J Med Inform 2015; 84: $141-148$

[6] Team RC. R: A language and environment for statistical computing. Vienna, Austria: R Foundation for Statistical Computing; 2014: Available from: https://cran.r-project.org/ Accessed 2018 out 11

[7] McMahon RQ, McCarthy EE, Hetzel S] et al. Focus on technology: How important is resolution in telecytopathology? Cancer Cytopathol 2014; 122: $546-552$

[8] Heimann A, Maini G, Hwang S et al. Use of telecytology for the immediate assessment of CT guided and endoscopic FNA cytology: diagnostic accuracy, advantages, and pitfalls. Diagn Cytopathol 2012; 40: $575-581$

[9] Kovalovsky A, Lenox R, Wang D et al. Use of dynamic telecytopathology for rapid on-site evaluation of endobronchial ultrasound-guided transbronchial fine needle aspiration biopsies. J Am Soc Cytopathol 2012; 1: S85-S86

[10] Hayashi T, Ishiwatari H, Yoshida M et al. Rapid on-site evaluation by endosonographer during endoscopic ultrasound-guided fine needle aspiration for pancreatic solid masses. J Gastroenterol Hepatol 2013; 28: $656-663$

[11] Ganc RL, Carbonari AP, Colaiacovo R et al. Rapid on-site cytopathological examination (ROSE) performed by endosonagraphers and its improvement in the diagnosis of pancreatic solid lesions. Acta Cir Bras 2015; 30: $503-508$

[12] Khurana KK, Graber B, Wang D et al. Telecytopathology for on-site adequacy evaluation decreases the nondiagnostic rate in endoscopic ultrasound-guided fine-needle aspiration of pancreatic lesions. Telemed J E Health 2014; 20: $822-827$

[13] Khurana KK, Rong R, Wang D et al. Dynamic telecytopathology for onsite preliminary diagnosis of endoscopic ultrasound-guided fine needle aspiration of pancreatic masses. J Telemed Telecare 2012; 18: 253259 